\title{
Relações de gênero e estética organizacional: sugestões para estudos sobre relações, cultura e desempenho
}

\author{
Gender relations and organizational aesthetics: suggestions for studies on relationships, \\ culture, and performance
}

Kenny Basso ${ }^{1}$

Jandir Pauli ${ }^{2}$

Verônica Paludo Bressan ${ }^{3}$

\section{Resumo}

Os estudos das relações de gênero e também sobre a estética organizacional podem ter amplas variações, por isso tais temáticas são presentes sob diferentes enfoques nas agendas dos pesquisadores. A consideração estética das organizações vem, ao longo dos anos, ganhando importância nas análises organizacionais, enquanto os estudos das relações de gênero emergem da diversidade de indivíduos que passam a compor as organizações. Este ensaio representa um esforço teórico para identificar intersecções entre essas temáticas para prover novos olhares sobre três diferentes trilhas dos Estudos Organizacionais: relações entre indivíduos e organização; cultura organizacional; e desempenho organizacional. Para tanto, serão apresentados os conceitos de relação de gênero nas organizações e estética organizacional, estudos sobre a relação entre os indivíduos e a organização, cultura organizacional e desempenho da organização, atentando para a discussão sobre possíveis pontos de intersecção entre tais temáticas. Para apresentar as sugestões para futuros estudos, foram englobados ensaios teóricos e trabalhos teórico-empíricos sobre relações de gênero e estética organizacional. Sendo assim, a contribuição deste ensaio recai sobre a agregação de duas literaturas (relações de gênero e estética organizacional) até então não usualmente relacionadas, mas que juntas podem prover contribuições relevantes e representativas aos estudos e análises organizacionais.

Palavras-chave: Gênero. Estética organizacional. Cultura organizacional. Desempenho organizacional.

Artigo submetido em 26 de julho de 2013 e aceito para publicação em 24 de fevereiro de 2014.

\section{DOI: http://dx.doi.org/10.1590/1679-39519753}

${ }^{1}$ Doutor em Administração pela Universidade Federal do Rio Grande do Sul - UFRGS; Professor no Programa de Pós-Graduação em Administração - PPGA da Faculdade Meridional - IMED. Endereço: IMED - Faculdade Meridional, Rua Senador Pinheiro, 304, Vila Rodrigues, CEP 99070-220, Passo Fundo - RS, Brasil. E-mail: bassokenny@gmail.com

2 Doutor em Sociologia pela Universidade Federal do Rio Grande do Sul - UFRGS; Professor no Programa de Pós-Graduação em Administração - PPGA da Faculdade Meridional - IMED. Endereço: IMED - Faculdade Meridional, Rua Senador Pinheiro, 304, Vila Rodrigues, CEP 99070-220, Passo Fundo - RS, Brasil. E-mail: jandir@imed.edu.br

3 Pós-graduanda em Marketing pela Faculdade Meridional - IMED; Supervisora Institucional na Faculdade Meridional - IMED. Endereço: IMED - Rua Senador Pinheiro, 304, CEP: 99070-220, Passo Fundo - RS, Brasil. E-mail: vebressan@gmail.com 


\begin{abstract}
Studies on gender relations and also on organizational aesthetics may have wide variations, thus these themes are present under various approaches in the agendas of researchers. The aesthetic consideration of organizations has, over the years, gained importance in organizational analysis, while studies of gender relations emerge from the diversity of individuals that become part of organizations. This essay represents a theoretical effort to identify intersections between these themes to provide new looks at three different tracks of Organizational Studies: relationships between individuals and organization; organizational culture; and organizational performance. For this, we will present the concepts of gender relation in organizations and organizational aesthetics, studies on the relation of individuals to the organization, organizational culture and organization performance, focusing on the discussion about possible intersection points between these themes. To present suggestions for further studies, we comprised theoretical and theoretical-empirical essays on gender relations and organizational aesthetics. Thus, the contribution of this essay lies on the aggregation of two literatures (gender relations and organizational aesthetics) hitherto not usually related, but which can together provide relevant and representative contributions to organizational studies and analyses.
\end{abstract}

Keywords: Gender. Organizational aesthetics. Organizational culture. Organizational performance.

\title{
Introdução
}

Dentre os diversos temas de pesquisa na área de Estudos Organizacionais, duas temáticas são pautas frequentes na agenda dos pesquisadores: a estética organizacional e as relações de gênero. Entretanto, poucos trabalhos utilizam conjuntamente os conhecimentos sobre estética organizacional e relações de gênero para a compreensão e análise de outros fenômenos organizacionais, tais como a cultura organizacional. Baseado nisso, este ensaio representa um esforço teórico para a identificação de intersecções entre os conhecimentos de estética organizacional e de relações de gênero nas organizações em três diferentes trilhas dos Estudos Organizacionais: relações entre indivíduos e organização; cultura organizacional; e desempenho organizacional.

Desse modo, a junção dos conhecimentos sobre estética organizacional e relações de gênero nas organizações pode auxiliar os pesquisadores a compreender, de forma mais aprofundada e ampla, como os indivíduos buscam (ou não) ligar-se às organizações, como desenvolvem emoções e sentimentos de pertencimento; como são geradas as histórias e os significados que influenciam a manutenção, reprodução ou alteração da cultura organizacional; e, por fim, como o conhecimento organizacional e a comunicação podem influenciar o desempenho da organização. Diante disso, por meio das sugestões para futuras pesquisas apresentadas ao longo do ensaio, procura-se instigar nos pesquisadores novas visões e diferentes percepções sobre os fenômenos analisados pelos Estudos Organizacionais.

Tanto o conhecimento sobre o papel da estética organizacional em diferentes fenômenos organizacionais quanto a implicação das relações entre e dentro dos gêneros em diferentes elementos da organização constituem, separadamente, importantes linhas de pesquisa nos Estudos Organizacionais. Corroborando esse aspecto, Taylor e Spicer (2007) identificam que o campo dos espaços organizacionais, por si só, está alcançando a maturidade, no entanto, os autores mencionam que notadamente ligações com outros temas também precisam ser feitas para garantir a amplitude do estudo dos espaços organizacionais. Mais especificamente, Tyler e Cohen (2010) informam que a interação entre o espaço da organização, ou seja, a visão da estética organizacional e a concepção de gênero, deve ser foco de futuros estudos, procurando elucidar as possíveis interações e os efeitos dessas interações em outros fenômenos organizacionais.

A abordagem estética faz com que as organizações também sejam compreendidas por meio dos seus artefatos, que, por sua vez, possuem um significado subjetivo e socialmente construído que pode interferir na vida organizacional dos indivíduos. Essa significação dos artefatos é construída por meio das percepções sensoriais das pessoas que, de algum modo, participam da organização ou com ela tem contato. Nesses 
termos, a estética organizacional pode fornecer novas formas de ver e conceber as organizações, tanto com o intuito de desenvolvimento teórico quanto com o interesse de revelar aspectos importantes aos gestores e a todos os trabalhadores envolvidos no desenvolvimento e na perenidade da organização. Com isso, a compreensão estética pode auxiliar a revelar histórias e experiências que sempre existiram nas organizações, mas que poucas vezes foram compreendidas ou mesmo alvo de compreensões pelos pesquisadores e interessados na análise das organizações.

Já os estudos das relações de gênero emergem da diversidade de indivíduos, aspecto que passa a compor as organizações. Sob o discurso da valorização e, por diversas vezes, da consideração da importância da igualdade a todos os indivíduos, "a literatura de gênero e organização tem elucidado uma ampla perspectiva e levantado um número de questões interessantes" (ALVESSON e BILLING, 1992, p. 77). Sendo esse um panorama literário sobre as relações de gênero, esses conhecimentos podem ser utilizados para aprofundar a compreensão das implicações existentes dessas relações em outros fenômenos organizacionais de constante importância para os pesquisadores, auxiliando, ainda, a reforçar cada vez mais o entendimento sobre o fenômeno das relações de gênero na academia de administração.

Baseado nisso, primeiro, são apresentados os conceitos de gênero e estética organizacional, sendo que, na sequência, as intersecções entre os conhecimentos de estética organizacional e relações de gênero nas organizações são discutidas diante das relações entre os indivíduos e a organização, a cultura organizacional e o desempenho organizacional. Convém ressaltar que, para a apresentação de sugestões para futuros estudos, ensaios teóricos e estudos empíricos clássicos e contemporâneos, provenientes tanto da literatura sobre relações de gênero nas organizações quanto da literatura sobre estética organizacional, foram utilizados, a fim de embasar as discussões propostas. A partir da compreensão dos estudos presentes nessas literaturas foi possível identificar pontos de intersecção que podem fornecer subsídios para a compreensão das relações entre os indivíduos e a organização, a cultura organizacional e o desempenho organizacional. Tais interações e sugestões para futuros estudos são desenvolvidas de forma teórica e explicitadas ao longo deste ensaio.

\section{Relações de Gênero nas Organizações}

Para definir gênero Mathieu (2009, p. 181) afirma que "gênero é um processo de categorização", onde o início se dá com a divisão entre homens e mulheres, não só pelas características físicas de cada um, mas, sim, pelas atividades executadas, pelos comportamentos, pelos objetos com que interagem e tarefas que cada categoria possui distintas das outras. Charlesworth e Baird (2007) consideram que gênero não deve ser considerado apenas "mulher", o que, segundo os autores, pode acabar marginalizando a equidade entre os gêneros, almejada pela maioria dos estudos. Dando continuidade à crítica, Charlesworth e Baird (2007) salientam que o estudo dos gêneros não ocorre no vácuo, por isso, é necessário compreender os amplos contextos e as situações nas quais os gêneros são envolvidos para que os estudos possam obter resultados plausíveis e com implicações reais à prática da igualdade entre os gêneros.

Na distinção entre sexo e gênero, Calás e Smircich (1999) colocam que sexo é definido pela constituição biológica dos indivíduos, enquanto que o gênero é definido pelas vivências e pelas interações, sendo um produto social. Andrade, Cappelle, Brito et al. (2002) citam que o estudo dos gêneros deve superar a barreira do biológico, ao passo que compreensões simbólicas e subjetivas devem ser consideradas nas análises, uma vez que os gêneros implicam uma construção compartilhada e coletiva. Visto isso, na sequência deste trabalho, será adotada a definição gênero, procurando instigar o pensamento sobre um processo de construção e sua dimensão relacional, e não sobre algo determinado (como na concepção de sexo). Nesse sentido, Fournier e Smith (2006) citam que, em vários estudos, é proposta uma posição contrária ao dualismo dos gêneros, uma posição fluida em que múltiplas identidades de gênero possam ocorrer entre femininos e masculinos. Todavia, os autores não compartilham essa posição e acreditam que uma nova espécie de fluidez 
possa ser colocada em pauta para estudos, que destitua a posição de gênero e não procure dentro da masculinidade ou feminilidade várias identidades. Esse entendimento de fluidez poderia permitir "mover para dentro e para fora do gênero, ao invés de entre as identidades de gênero" (FOURNIER e SMITH, 2006, p. 160).

Sob uma perspectiva masculina dos estudos de gênero, Freedman e Phillips (1988) colocam que a pesquisa sobre os gêneros nas organizações despertou em três principais frontes: 1) sobre o impacto econômico das mulheres no trabalho; 2) modelos para explicar a presença ou ausência de diferenças de gênero nos comportamentos organizacionais; 3) mudanças nos valores sociais, aspectos legais e o aumento de mulheres ingressando no ambiente organizacional. Segundo Cappelle, Brito, Melo et al. (2006), devido às alterações nas dinâmicas empresariais nos últimos anos, têm sido frequente os estudos que procuram debater questões sobre gênero no ambiente organizacional.

Também acerca das discussões, Murgia e Poggio (2009) colocam que a maioria dos estudos sobre gênero e organizações tem sido concentrada na experiência feminina vivenciada e nos aspectos que contribuem para a construção da feminilidade, buscando geralmente contrapontos na masculinidade e nas experiências que os homens têm nas organizações. Porém, alguns estudos vêm buscando não só contrapontos entre os gêneros, mas, também, diferenças que possam existir dentro dos gêneros, como, por exemplo, no estudo de Holvino (2010), em que diferenças de classe social e raça são confrontadas dentro do gênero feminino, provocando discussões acerca do impacto de como as diferenças de raça (cor) e classe social podem ser responsáveis pelas diferentes formas de pensamento e crítica aos movimentos femininos organizacionais. Com o mesmo raciocínio, Mathieu (2009) afirma que a relação entre os gêneros é uma parte ativa e importante na organização, sendo que não devem ser consideradas apenas as relações entre homens e mulheres, por exemplo, mas, também, as existentes entre homens (masculino) e homens (masculino) e entre mulheres (feminino) e mulheres (feminino).

Ainda que várias considerações e proposições sejam apresentadas nos mais diversos estudos, Mathieu (2009) considera que ainda há um gap entre a prática e a consciência discursiva sob a inclusão dos gêneros no ambiente organizacional. Por vezes, a conduta prática é contrária ao intencionado e ao almejado antes da ação. Diante disso, Murgia e Poggio (2009) colocam que a característica dinâmica, ou seja, a constante mudança dos gêneros e das suas relações pode ser uma causa dessa lacuna, que também pode ser influenciada pelas dinâmicas e processos históricos da sociedade, bem como pelas características físicas e estéticas dos espaços de trabalho.

Embora essa vertente busque estabelecer certo dualismo no que tange ao entendimento de gênero, classificando-o entre homens e mulheres, as discussões sobre gênero também englobam estudos que procuram revisitar o significado de gênero e propor novas discussões. Nesse espectro, não se pode deixar de considerar a evolução da categoria gênero e o ambiente em que a crítica se desenvolveu, especialmente no Ocidente. Scott (1995) faz uma análise, desde os primeiros estudos feministas, procurando dar ao conceito de gênero o status de categoria analítica para compreender seu funcionamento nas relações sociais. A autora resume os estudos em três diferentes perspectivas: a primeira é inteiramente feminista, a segunda inteiramente marxista e comprometida com a crítica feminista, enquanto a terceira é dividida entre o pósestruturalismo francês e as teorias anglo-americanas de relação do objeto (object-relation theories).

Segundo Scott (1995), os estudos feministas, que surgiram nos anos 1970, procuraram escrever "uma história das mulheres", seguindo a tradição inaugurada com as categorias de classe e raça. Esses estudos, em geral descritivos, foram importantes porque ampliaram a noção de história para além da guerra, do Estado e da economia. A grande contribuição desses estudos foi alargar as noções tradicionais do que é importante no sentido histórico, isto é, reescrever a história para além da economia e da política. No entanto, ao passo que a tradição reconheceu a história das mulheres, como consequência, houve a impressão de que essa história está separada daquela dos homens, que está associada à política e à economia, enquanto a das mulheres está associada ao sexo e à família. 
Além disso, a opção por estudar "as mulheres" conferiu aos primeiros estudos uma conotação política, na medida em que se apoiava na libertação das mulheres em relação às regras impostas pelo patriarcado. Por isso, os estudos recentes começaram a utilizar a categoria de gênero como sinônimo de "organização social das relações entre os sexos", procurando explorar no conceito uma dimensão relacional entre os sexos, especialmente as "definições normativas da feminilidade" (SCOTT, 1995, p. 72). Com isso, gênero virou sinônimo de mulheres. Falar em gênero, ao invés de mulheres, tinha por ideia dar uma conotação mais neutra e objetiva, criando uma terminologia mais aceita na ciência (em oposição aos estudos supostamente ruidosos e da política feminista). Além disso, o termo foi utilizado para sugerir informações sobre as mulheres, mostrando que toda informação sobre elas é produzida a partir e em relação ao mundo dos homens. Essa perspectiva reforçou a ideia de que um sexo tem pouco ou nada a ver com o outro e a categoria de gênero passa novamente a ser uma "categoria social imposta sobre um corpo sexuado" (SCOTT, 1995, p. 72).

Scott (1995) insiste na ideia de que a questão a ser discutida é de que a história das mulheres foi construída na sua diferença (oposição) em relação à história dos homens. Por isso, os esforços no atual contexto do debate de gênero devem explorar o elemento relacional e fixo que marcou a tradição da utilização dos conceitos. Para a autora, "temos necessidade da rejeição do caráter fixo e permanente da oposição binária, de uma historicização e de uma desconstrução genuínas dos termos da diferença sexual" (SCOTT, 1995, p. 84).

A perspectiva/definição da autora pode ser resumida a partir de uma conexão integral entre duas proposições: 1) gênero é um elemento constitutivo de relações sociais baseadas nas diferenças percebidas entre os sexos; e 2) gênero é uma forma primária de dar significado às relações de poder (SCOTT, 1995). Com isso, procurou superar a noção de que gênero é constituído para além do ambiente doméstico e do parentesco, transpassando as relações econômicas e políticas, presentes em instituições sociais como o mercado de trabalho, da educação e do sistema político. Em outros termos, os significados de gênero e de poder são construídos de maneira recíproca, o que permite elevar o conceito ao status de categoria analítica de organização das relações sociais, sem perder a perspectiva normativa implícita nesse debate.

No plano das relações de poder, o francês Pierre Bourdieu (1930-2002) desenvolveu dois conceitos importantes para ambientação da problemática aqui proposta: o conceito de habitus ${ }^{4}$ e de dominação masculina. Seu ponto de partida são os estudos realizados nos anos 1950 e 1960, na sociedade Cabila ${ }^{5}$, nos quais procurou evidenciar que a estrutura falonarcísica encontrada nesses povos, baseada em estruturas cognitivas e sociais introjetadas nos indivíduos, também está presente nas sociedades ocidentais.

A divisão dos gêneros inscrita na ordem social das coisas, a divisão dos dias, do calendário de atividades rurais, de espaço, bem como a oposição entre a casa e a assembleia, todas as divisões objetivas se veem inscritas nos corpos, na forma de disposições e se tornam princípios subjetivos de visão, categorias cognitivas através das quais os indivíduos veem e constroem o mundo como realidade significativa, viva. Tendo se originado do mundo, tais esquemas de percepção estão de acordo com a ordem objetiva das coisas e nos inclinam a tomar o mundo como dado. Essa concordância espontânea entre as estruturas sociais e as estruturas cognitivas - quando ocorre - é a base da experiência dóxica da dominação masculina como inscrita na natureza das coisas, invisível, não questionada (BOURDIEU, 1999, p. 18).

${ }^{4}$ Habitus é um sistema de esquemas de percepção e apreciação dos indivíduos que funcionam a partir de estruturas cognitivas e avaliatórias adquiridas por meio da experiência no mundo social, funcionando como mediadores entre os condicionamentos exteriores e sua subjetivação. É o habitus que permite o funcionamento da violência simbólica, uma vez que ela só se efetiva quando as estruturas dominantes são incorporadas (subjetivadas) pelos dominados.

${ }^{5}$ Sociedade camponesa, originária da tribo Kabiles, que habitava as montanhas Atlas, no norte da África. Para Bourdieu, essa sociedade estava estruturada em classificação a partir de um sistema de oposições (masculino/feminino, leste/oeste, quente/frio) que se expressava, por exemplo, na divisão sexual do trabalho. 
Em outros termos, a divisão entre os sexos parece estar na ordem natural das coisas, nos esquemas mentais, nos corpos e no mundo objetivado das coisas (casa, empresa etc.), compondo o habitus corrente. Nesse sentido, o pensar sociológico deve estar orientado pela recusa a uma visão androcêntrica e por uma postura política disposta a travar "lutas cognitivas", resistindo à ideia de divisão sexual entre homens e mulheres. Bourdieu acredita que esses esquemas mentais estão presentes, inclusive, em algumas teorias feministas.

É nesse plano que se desenvolve a violência simbólica, compreendida como adesão do dominado ao dominante para se ver e avaliar ou para ver e avaliar os dominantes, resultante da subjetivação das classificações "naturalizadas" de que seu ser social é produto (BOURDIEU, 1999, p. 47). Nesse sentido, mulheres são objetos que circulam no mercado de bens simbólicos a partir de um papel fixo em sua ação social, ao passo que supervaloriza a estrutura masculina.

A principal contribuição de Bourdieu (1999) e Scott (1995) para este estudo é lançar luzes para compreender gênero a partir do ambiente das empresas, tendo por objeto analítico a questão da estética organizacional. Nesse sentido, cabe perguntar se as organizações empresariais podem ser compreendidas como espaços de reprodução do habitus masculino. Adotando-se a terminologia de Bourdieu (1999) e conferindo às organizações o status de "campo", dotado de um habitus específico em que as mulheres são consideradas "recém-chegadas", seria possível prever, por parte delas, dois tipos de comportamento: 1) adaptar-se às normas e agir dentro do modelo proposto ou 2) procurar subverter a ordem por meio do enfrentamento às estratégias de dominação. Em geral, as organizações mantêm programas pedagógicos de acolhimento e adaptação dos novos integrantes, evitando os custos de eventuais tentativas de desestruturação do habitus estabelecido. Quais são os símbolos da dominação masculina e como perceber esse habitus nas rotinas e nos procedimentos da organização empresarial? É possível reconhecer estratégias de violação do habitus na perspectiva relacional de gênero capaz de romper com a identidade masculina cristalizada nas organizações empresariais? Para instigar pontos referentes a essas questões, apresenta-se, na seção seguinte, uma discussão acerca de estética organizacional para, na sequência, evidenciar as possíveis intersecções entre as duas literaturas.

\section{Estética Organizacional}

Inicialmente, para definir estética organizacional, Taylor e Hansen (2005) citam que a estética concerne ao conhecimento gerado a partir das experiências sensoriais do indivíduo, que, por sua vez, engloba os sentimentos e o raciocínio sobre as vivências individuais. A estética organizacional, segundo Martin (2002), requer uma experiência sensorial e uma reação a essa experiência, o que confere à estética organizacional um papel interativo com os indivíduos e não apenas um papel passivo no processo de construção da organização. Para Strati (1992) a estética organizacional é, ao mesmo tempo, uma linha de estudo como um tópico e um objeto de análise, podendo ser vista, de acordo com Gagliardi (2001), como um conhecimento relegado em prol do desenvolvimento do conhecimento lógico-científico, ou seja, objetivo, uma vez que o conhecimento da estética organizacional envolve a subjetividade inerente à percepção e aos sentidos conferidos aos artefatos organizacionais.

Enquanto as experiências de Hawthorne (meados de 1920) já enfocavam os elementos físicos da organização (p. ex. iluminação), a pesquisa sobre a estética organizacional procura confrontar os sentidos dos indivíduos com seu senso de corporalidade e da organização como um todo (MACK, 2007). Diante disso, Witz, Warhurst e Nickson (2003) citam que estética e organização são inseparáveis, já que a estética está presente em toda organização, por meio dos artefatos que constituem sua forma de expressão, sua identidade. Mack (2007) coloca que a estética organizacional atenta, dentro da fábrica ou da organização, para os elementos sensíveis, que podem ser vistos, tocados, ouvidos, cheirados ou provados (pelo paladar). Consequentemente, categorias como cômico, trágico, sagrado, feiura e beleza podem ser encontradas nas descrições da estética organizacional, o que reflete a vida das pessoas na organização. Strati (1992) coloca que a categoria da 
beleza pode ser útil nas análises e compreensões das organizações, pois ela pode revelar significados profundos, podendo ser utilizada, também, em uma ampla gama de situações para o entendimento da organização.

Embora, segundo Taylor e Hansen (2005), a estética organizacional se confunda, por diversas vezes, com a beleza das organizações ou a busca por ambientes mais belos para a execução do trabalho, o lado grotesco ou bruto parece ser tão relevante quanto para a compreensão da estética organizacional, explicitando como ela pode ser imprescindível aos Estudos Organizacionais.

Para Taylor e Hansen (2005, p. 1226) os questionamentos sobre estética organizacional aprofundam o "conhecimento das organizações por prover uma nova epistemologia, critérios para acessar os julgamentos e tomadas de decisões dos membros, significados, conexões e prover categorias para dados sensoriais". Nesse sentido, uma maior compreensão da estética organizacional parece não ser importante somente para o desenrolar dos estudos acadêmicos, mas, também, para a prática e o aprimoramento da gestão das organizações. Com o mesmo raciocínio, Martin (2002) afirma que o entendimento da estética da organização pode ser feito por meio das reações que os indivíduos apresentam em relação a ela.

No entanto, para observar essas reações à estética organizacional, Martin (2002) revela que uma das principais dificuldades dos estudos é separar os próprios julgamentos e percepções sensoriais do pesquisador daquelas que estão sendo sentidas pelos indivíduos que "vivem" a organização, para tanto, segundo a autora, deve-se adotar uma espécie de princípio positivo de neutralidade, ciente de que a plena neutralidade inexiste. Para Gibb (2004), a pesquisa sobre estética organizacional assume a forma de uma pesquisa etnográfica, na qual a apreensão das crenças, valores, práticas e artefatos da organização são importantes para a compreensão da lente estética que os indivíduos podem possuir sobre o local em que trabalham.

Convém ressaltar que, nesse contexto, os artefatos não são apenas elementos decorativos, mas, sim, considerados atores da estética organizacional que possuem, em sua individualidade, a representação do todo da estética da organização. Cox e Minahan (2005) revelam que a falta de artefatos também pode ser compreendida como uma decoração organizacional, podendo trazer significados tanto quanto a presença dos artefatos naquele espaço.

Segundo o mesmo raciocínio, para Weggeman, Lammers e Akkermans (2007), a estética não se encontra somente na organização, pois, segundo os autores, organizações mais bonitas e com produtos ou ofertas esteticamente mais atraentes podem ter um desempenho melhor no seu ambiente competitivo. Dessa forma, os mesmos autores sugerem que, para que os produtos sejam bonitos, sob o prisma da estética, os processos organizacionais que os geram também devem ser, sendo que, para tanto, a satisfação e felicidade dos trabalhadores que neles atuam deve existir, o que, por sua vez, implica um espaço de trabalho esteticamente agradável, que os faça se sentir bem. Para obter tal espaço, dois aspectos são essenciais: a sensibilidade estética e a educação para a estética, ou seja, os gestores devem possuir conhecimento sobre a estética organizacional e seu impacto no desempenho da organização, devendo, também, ter sensibilidade quanto às condições e aspectos que devem ser providos para tal.

Baseado nisso, para compreender a complexidade organizacional, é relevante estudar as coisas materiais e como elas possuem impacto na ação humana para que se possa ter um retrato mais completo do fenômeno organizacional a ser estudado, uma vez que parece ilusório imaginar que o indivíduo não tenha relação com as coisas materiais, sofrendo influência destas e influenciando-as. Sob esse aspecto, a compreensão do que essas coisas materiais significam para o indivíduo (positiva ou negativamente) parece ser relevante para a melhor compreensão do funcionamento da organização, habilitando o pesquisador a alcançar uma profundidade de análise do fenômeno, a qual lhe permite uma compreensão mais assertiva da complexidade do fenômeno organizacional em análise. 
Na sequência do estudo, a utilização dos conhecimentos sobre as relações de gênero e estética organizacional são discutidos dentro da possibilidade de compreensão das relações entre os indivíduos e a organização, da cultura organizacional e do desempenho organizacional.

\section{Relações Entre os Indivíduos e a Organização}

Os indivíduos imersos em uma organização por meio de relações de trabalho possuem, de algum modo, relação dual com a organização, interferindo nela e sofrendo sua interferência em suas vidas. Em outras palavras, a relação de trabalho dos indivíduos faz com que sejam despertados sentimentos em relação à organização, que podem ser tanto de pertencimento e de construção de uma relação duradoura quanto de repulsa e de vontade de deixar o local de trabalho o mais breve possível. Desse modo, os indivíduos constroem subjetivamente conexões com a organização onde trabalham, as quais podem sofrer a influência das condições estéticas do espaço de trabalho, bem como das relações de gênero estabelecidas na organização.

Nisso, a compreensão do sistema patriarcal pode auxiliar o entendimento das variáveis que circundam a construção das conexões masculinas com a organização (MURGIA e POGGIO, 2009). Para Alvesson e Billing (1992), a consideração de que ideologias, valores e padrões de comportamentos masculinos dominam as organizações pode ser um alento para o estudo do gênero na organização, uma vez que a perspectiva do gênero pode contribuir para o entendimento das ligações entre indivíduos e organização, que, por sua vez, pode ser vista como "masculina" ou masculinamente dominada.

Segundo Andrade, Cappelle, Brito et al. (2002), também é aceito que o ambiente de trabalho pode ser compreendido como um espaço que implica a construção de significados tanto para os gêneros femininos quanto para os masculinos. No entanto, os autores referem que a pluralidade deve ser compreendida e respeitada, ao invés de procurar considerar todos os gêneros como iguais, isto é, não se deve considerar a igualdade, mas, sim, compreender e respeitar as distinções. Diante disso, diferentes significados do ambiente de trabalho podem emergir de diferentes gêneros, que, por sua vez, têm contato com diferentes objetos. Conceber as organizações como espaços tangíveis carregados de artefatos permite entender a experiência estética vivenciada pelos indivíduos que fazem parte da organização, ao mesmo tempo que habilita o entendimento dos diversos significados que podem advir e ser atribuídos ao lugar, à realidade física em que se constitui a organização (GAGLIARDI, 2001). Baseado nisso, pode-se compreender os motivos pelos quais os indivíduos sentem-se conectados ao seu espaço de trabalho.

Sob esse aspecto, a estética organizacional representa uma forma de construção de conexões, ou seja, os artefatos provocam sentimentos de pertencimento a determinado local, podendo interferir nos agrupamentos dos indivíduos e também nas suas relações sociais nesses agrupamentos (TAYLOR e HANSEN, 2005). No mesmo sentido, Rafaeli e Vilnai-Yavets (2004) colocam que os sentimentos que os indivíduos têm com os artefatos que compõem a organização são os mesmos que possuem com a organização, sendo que os artefatos, dessa forma, atuam como itens de representação da organização, por meio dos quais os trabalhadores podem expressar seus sentimentos e emoções, por meio da criação de sentido.

Nisso, Weick (1995) cita que os objetos da organização podem atuar como pontos centrais para a criação de sentido, que envolve mais de um indivíduo, atuando, portanto, como um item integrador do processo contínuo de criação de sentido. Sendo assim, a criação de sentido do artefato também pode estar ligada à criação de sentido da organização, que ocorre de forma coletiva (RAFAELI e VILNAI-YAVETS, 2004; WEICK, 1995), podendo intervir e auxiliar na construção das relações entre e dentro dos gêneros. No mesmo sentido, Witz, Warhurst e Nickson (2003) citam que os elementos estéticos são utilizados pelos empregados para se expressar e desenhar uma imagem de si no ambiente organizacional. Nesse senso de conexão, Mack (2007) coloca que é natural ao indivíduo procurar formas de conectar-se com o lugar onde se encontra, para 
que possa conviver com aquele ambiente, por isso, usar determinadas ferramentas pode representar a conexão com esse ou aquele gênero.

Essa utilização dos objetos para construir uma conexão com o ambiente pode ser feita de diferentes formas e ir ao encontro das colocações de Shaw e Cassell (2007), que advogam que as diferenças de gênero não são decorrentes dos próprios gêneros, mas, sim, de outras variáveis, como os artefatos que são utilizados por cada gênero, podendo estes, portanto, ser utilizados para diferenciação entre os gêneros. Assim, ao procurar usar os artefatos para se conectar ao ambiente, cada gênero pode estar criando suas próprias diferenciações. Diante disso, Cairns (2002) coloca que a estética organizacional, ou seja, o espaço físico, pode tanto fornecer símbolos que transmitam uma ideia de pertencimento aos empregados quanto ter uma conotação negativa desagradável, onde a organização pode ser vista como uma estrutura de controle, já que reúne diversos indivíduos no mesmo espaço físico. Tal como ocorre quando os indivíduos criam um sentido para as coisas que lhes é benéfico ou contribui para que estes se sintam bem na organização, os sentidos também podem enfocar aspectos negativos, tal como a opressão gerada pelas coisas materiais, fazendo com que os empregados sintam-se insatisfeitos ou que tenham sentimentos negativos decorrentes das coisas materiais que compõem a organização. Essa concepção negativa ou opressora pode ser uma das causas da subestimação e discriminação das mulheres no âmbito do trabalho, uma vez que os artefatos podem estar sendo utilizados com o intuito (conscientemente ou não) de gerar percepções opressoras em um gênero (feminino) e libertadoras ou empáticas em outro (masculino).

Ainda quanto às mulheres nas organizações, Fournier e Smith (2006) citam que, para atingir posições de sucesso no ambiente organizacional, as mulheres suprimem seu lado feminino e adotam comportamentos e expressões masculinas, passando a adotar posições estéticas e percepções masculinas para se conectar com a organização. Segundo Andrade, Cappelle, Brito et al. (2002), a convivência das mulheres com os homens torna-se a saída mais plausível para que as mulheres construam suas identidades, sem relegar ou desconsiderar a identidade na qual estão imersas, e, com isso, conseguir construir uma relação mais feminina com a organização.

Baseado no exposto, apresenta-se a primeira sugestão de estudo:

\section{Sugestão 1: Conjuntamente, os conhecimentos sobre a estética organizacional e as relações de gênero podem propiciar uma compreensão sobre a complexidade que envolve a conexão dos indivíduos com a organização.}

Em outro aspecto, as reações à estética organizacional são inerentemente imbuídas de um aspecto relacional, ou seja, as relações sociais e práticas entre os indivíduos na organização podem denotar uma direção para as reações à estética organizacional, assim como a estética organizacional pode interferir na construção das relações sociais (MARTIN, 2002). Dessa forma, se as relações sociais estão imbuídas de masculinidade é possível que as reações à estética também sejam reações masculinizadas, exceto quando, conforme colocam Fournier e Smith (2006), a feminilidade não é oprimida ou mascarada pela masculinidade, fazendo com que ela revele seu real sentido, sua autenticidade, do contrário, a masculinidade continuará sendo dominante.

Diante disso, as mulheres na organização percebem-se mais semelhantes aos homens quanto mais elevada a posição ocupada, ou seja, em altos postos organizacionais as mulheres afirmam que não percebem diferenças entre suas características e as características de masculinidades por elas elencadas (LEDET e HENLEY, 2000), pois podem estar compartilhando os mesmos elementos estéticos e significados masculinos que tradicionalmente permeiam os cargos e relações sociais mais elevadas da organização. Rafaeli e VilnaiYavets (2004) atestam que a estética organizacional também é diretamente responsável pela geração de emoções no indivíduo, que terão conexões diretas com as emoções sentidas por este em relação à organização como um todo, construindo, assim, um senso de conexão emocional. Essas emoções, 
decorrentes das construções e coisas materiais presentes na organização, podem ser encaradas como forma de controlar os indivíduos (CAIRNS, 2002), uma vez que os mantêm juntos e agrupados, criando um senso de unidade, o que facilita o controle por parte da organização. Desse modo, as reações emocionais também podem indicar a relação do indivíduo a determinado gênero, implicando, com isso, um maior conhecimento da conexão existente entre os gêneros e a organização, o que ocorre por meio dos artefatos estéticos que cada gênero utiliza ou percebe como seus (identificação).

Segundo Strati (1992, p. 575), "a estética da vida organizacional não é observável em alguma forma pura", sendo que ela pode ser compreendida por meio da legitimação a ela conferida e também por meio das vozes e relações entre os membros que a ela são submetidos. Nesse sentido, as vozes dos membros da organização podem ser compreendidas por meio do uso, da teatralidade e do cultivo da aparência dada pelos empregados no espaço de trabalho, não se tratando apenas de suas vestimentas, mas, também, de como seu local de trabalho é mantido, quais artefatos estão presentes, como isso interfere nas suas ações e relações com os outros e como suas ações modificam ou alteram essa estética (WITZ, WARHURST e NICKSON, 2003).

Taylor e Hansen (2005) colocam que compreender as visões sobre a estética organizacional, se feia ou bonita, por exemplo, pode auxiliar a atrair e manter os diferentes gêneros no ambiente de trabalho, uma vez que as pessoas são atraídas pelo que julgam bonito e tendem a se afastar do que percebem como feio, o que incorre em diferentes tipos de emoções. Todavia, segundo Martin (2002), os julgamentos estéticos não se referem apenas à coisa que se está julgando, mas levam em consideração a interpretação e a subjetividade inerente às percepções individuais, o que pode intervir no processo de compreensão dos significados que, por sua vez, é distinto para cada gênero.

Com isso, a segunda sugestão, baseada na intersecção dos conhecimentos sobre estética organizacional e relações de gênero, é apresentada:

\section{Sugestão 2: A junção dos conhecimentos sobre a estética organizacional e sobre as relações de gênero pode auxiliar a compreender como o sujeito desenvolve seu sentimento de pertencimento à organização.}

Com o exposto, é possível identificar que as conexões dos indivíduos com a organização perpassam as relações entre os gêneros existentes e também as interações que estes têm com os artefatos que representam a estética da organização. Essas conexões são desenvolvidas, por vezes, de forma subjetiva, envolvendo emoções, identificação entre membros da organização, questões socioculturais, externas à organização, e mesmo aspectos da formação do indivíduo. Dessa forma, a compreensão de como essa interação entre indivíduos e organização ocorre pode trazer conhecimentos teóricos relevantes para a compreensão dos motivos pelos quais os indivíduos se identificam ou não com a organização, apresentando indícios sobre os motivos que os levam a permanecer muitos e muitos anos ou apenas alguns dias.

\section{Cultura Organizacional}

Segundo Deshpande e Webster (1989, p. 4), a cultura organizacional pode ser compreendida como "um padrão compartilhado de crenças e valores que ajudam os indivíduos a entender o funcionamento organizacional e também provê a eles normas para o comportamento na organização". Smircich (1983) identificou duas linhas distintas para a definição de cultura organizacional: a primeira, tratando-a como uma variável independente, resposta à sociedade à qual pertence, sendo absorvida e inserida na organização por meio dos membros dessa sociedade, sendo, dessa forma, externa à organização. A segunda definição apresenta a cultura organizacional como uma variável interna, na qual as organizações são, elas mesmas, 
responsáveis pelo fenômeno da formação de determinada cultura, produzindo produtos culturais, como lendas e cerimônias. Segundo Morgan (1996), não é possível identificar a cultura como algo imposto com base em uma situação social, uma vez que esta será desenvolvida com base nas interações sociais.

A partir dessas definições, levando em consideração a possibilidade de absorção de características da sociedade em que está inserida e, mais ainda, de produzir uma cultura própria, é possível inferir que grupos menores podem desenvolver espécies de subculturas diferentes dentro de uma mesma organização. Pode-se identificar, dessa forma, que as diversas configurações de gênero podem influenciar a criação dessas subculturas ou culturas independentes, em termos de explicação de comportamento e normatização, daquela tida como a cultura de toda a organização. Nesse sentido, Morgan (1996) afirma que a cultura organizacional deve ser compreendida como um aspecto vivo, por meio do qual as pessoas podem ver e rever os contextos nos quais vivem.

Em relação à estética, para Cox e Minahan (2005), o estudo dos objetos e da decoração organizacional tem uma proximidade com os estudos da cultura organizacional, uma vez que o design do interior, o tamanho do local de trabalho e as vestimentas dos empregados, por exemplo, podem indicar o que é importante para a organização e o que é cultuado dentro dos significados organizacionais. As vestimentas, nesse caso podem ser utilizadas para projetar a imagem desejada da organização nos empregados e também na comunidade onde ela está inserida (WITZ, WARHURST e NICKSON, 2003). Em relação à cultura, Gibb (2004) coloca que a estética organizacional, por meio de um enfoque arqueológico, pode envolver a identificação e compreensão dos símbolos e valores que, presentes na organização, representam sua cultura. Esses símbolos e valores, interpretados sob uma perspectiva estética, podem auxiliar na compreensão mais ampla da cultura organizacional, uma vez que não representam apenas artefatos, mas, sim, histórias que podem ser contadas e interpretadas, a fim de criar um sentido para a cultura organizacional.

Essas histórias podem ser as responsáveis pela construção de uma cultura que separa os gêneros. Nesse sentido, Shaw e Cassell (2007), ao estudar o âmbito acadêmico, colocam que a cultura masculina ainda gera barreiras para a entrada das mulheres no trabalho, posição esta também defendida pelo feminismo radical. No entanto, as mesmas autoras colocam que altos níveis de direcionamento e ambição das mulheres pode ser um sinal de que mudanças sobre a importância do trabalho na construção da identidade feminina estão cada vez mais evidentes. Murgia e Poggio (2009) citam que contar histórias sobre as relações familiares ou sociais pode atuar como um mecanismo preventivo e reforçador da cultura masculina dominante, isto é, a atividade de contar histórias pode atuar como um reprodutor das relações existentes entre os gêneros em determinado contexto. Em outro sentido, a atividade de contar histórias também pode ser um mecanismo que auxilia a reverter a cultura masculina dominante e a reduzir as diferenças entre os gêneros, quando direcionada para tal (MURGIA e POGGIO, 2009). Cappelle e Netto (2001), ao analisar as representações sociais entre policiais militares, encontraram que, embora a cultura organizacional dessa instituição possa estar em mudança, ainda há uma prevalência da dominação masculina.

Diante dessa prevalência da cultura masculina, deve-se colocar também que, em cada contexto, pode ser encontrada uma relação diferente entre os gêneros, devido às múltiplas realidades que advém da diversidade de linguagens, temporalidades e percepções dos indivíduos que compõem essas diversas realidades (CAIRNS, 2002). Nesse sentido, encaixa-se também o conceito de beleza da estética organizacional que não pode ser dissociado da percepção que reside na mente humana, sendo desta dependente, uma vez que a criação de sentido ocorre em nível individual, assim como coletivo (WEICK, 1995).

Além disso, Gagliardi (2001) afirma que a construção do eu, ou de uma identidade, tem relação com as coisas materiais que são utilizadas pelos indivíduos, as quais podem refletir quem estes são, qual é sua personalidade. As pessoas também parecem recorrer aos objetos para garantir uma sustentação da identidade que buscam construir ao longo do tempo. Sob esse aspecto, a estética organizacional também parece ser composta por coisas que os indivíduos carregam para o ambiente de trabalho, por meio das quais buscam evidenciar sua identidade e fornecer pistas para os outros, instigando com isso, a construção de ligações entre 
os indivíduos do mesmo gênero, ou de outro gênero, por meio dos significados que as coisas materiais podem ter para cada grupo.

Os artefatos carregados para o ambiente, que atuam como reforçadores da construção de uma identidade pessoal, também agem como intervenientes na cultura organizacional, ao passo que constituem os símbolos que garantem o significado da cultura. Diante disso, também convém destacar que cada gênero possui uma noção de beleza distinta e, por isso, a combinação entre as relações de gênero e a estética organizacional, pode contribuir para o estudo da cultura organizacional. Sendo assim, apresenta-se a seguinte sugestão de estudo:

\section{Sugestão 3: A intersecção dos estudos de estética organizacional e de relações de gênero nas organizações permite compreender como as histórias, significados, simbolismos e habitus masculino apresentados na organização influenciam a cultura organizacional.}

Em outro aspecto, baseado na capacidade de reprodução da cultura, as estruturas organizacionais, segundo Alvesson e Billing (1992), podem sofrer interferência da socialização e construção dos gêneros, assim como a produção dos símbolos, tarefas e funções organizacionais também podem ser influenciadas. Os mesmos autores afirmam que o simbolismo decorrente dos gêneros pode incorrer no benefício de apenas uma parte, podendo acarretar, em casos mais extremos, a saída do outro gênero da organização. Além disso, Tiessen (2004) coloca que as normas sociais e as percepções culturais, ao impactar as programações atitudinais dos gêneros, acabam impactando também a constante formulação da organização e a relação dos gêneros com a organização como um todo. Corrêa, Gontijo, Assis et al. (2007) ao analisar jornais internos de organização, constataram que essas mídias podem servir como mecanismos de disseminação dos modos de agir dentro da organização, que são diferentes para homens e mulheres. Enquanto as publicações dirigidas às mulheres adotam uma postura mais cotidiana, ligada à vida doméstica, às relações sociais, aos sonhos e desejos das mulheres contemporâneas; as publicações masculinas transmitem uma postura mais racional, frisando a competitividade e a disposição dos indivíduos masculinos no ambiente de trabalho (CORRÊA, GONTIJO, ASSIS et al., 2007). Nesse sentido, tais publicações acabam refletindo e reproduzindo as diferenças já existentes na sociedade, além de reforçar a atuação dos artefatos estéticos sobre os gêneros e a cultura que impera.

Assim, as diferenças de socialização e percepções que os gêneros possuem acabam externalizando-se por meio das modificações e criações de sentido para os artefatos organizacionais, revelando outra intersecção entre os conhecimentos de estética organizacional e relações de gênero, apresentada na sugestão de estudo a seguir:

\section{Sugestão 4: A junção dos conhecimentos sobre estética organizacional e sobre as relações de gênero nas organizações pode fornecer indicativos de como a cultura organizacional é mantida, reproduzida ou alterada, bem como as ações de resistência e adaptação das organizações às novas realidades.}

Por fim, o estudo sobre a cultura organizacional pode ser um ponto de tangência entre os estudos de gênero e os estudos sobre a estética das organizações. Aliado a isso, sugere-se que, em conjunto, concepções estéticas e compreensões das interações e diversidades dos gêneros podem evidenciar uma maior profundidade nas interpretações das culturas organizacionais. Especificamente, acredita-se que tais intersecções podem identificar simbolismos e habitus masculinos nas organizações, especialmente suas estratégias de reprodução, bem como, as ações de resistência e adaptação destas às realidades em constante mudança. Eccel (2010) menciona que os novos estudos devem procurar compreender, além das experiências dos gêneros, os 
aspectos coletivos e culturais, possibilitando, assim, uma compreensão acerca dos elementos que compõem a significação masculina e feminina, que determinarão as experiências subjetivas dos indivíduos no ambiente de trabalho. Isso pode prover tanto um ganho teórico ao estudo das organizações como, também, uma forte implicação prática para o entendimento de determinados fenômenos culturais nas organizações.

\section{Desempenho da Organização}

O desempenho da organização em seu ambiente pode ser diretamente influenciado pela comunicação interna e pelos conhecimentos que permeiam e são gerados dentro dela. Nesse aspecto, tanto a comunicação quanto o conhecimento serão utilizados como termos influenciadores do desempenho organizacional neste ensaio. Assim, os artefatos podem ser utilizados tanto como uma forma de comunicação entre os indivíduos quanto para gerar conhecimento tácito para que esses possam "viver" dentro da organização e, também, contribuir para o desempenho aceitável ou desejável desta em seu ambiente externo. Já as relações entre os gêneros incorrem claramente em processos comunicativos, sejam eles explícitos ou implícitos na relação, que, por sua vez, também gera conhecimentos necessários para que o indivíduo se torne e permaneça, efetivamente, sendo membro da organização.

Dentro das indústrias, as diferenças de gêneros como antecedentes do desempenho organizacional foram estudadas sob diversos prismas (SHAW e CASSELL, 2007). No passado, as mulheres eram vistas como intelectualmente e fisicamente inferiores aos homens, o que criou barreiras para sua entrada no mercado de trabalho gerando, segundo Shaw e Cassell (2007), ainda mais discriminação quando o gênero feminino começou a adentrar a esfera de trabalho nas organizações. Para Mathieu (2009) o gênero é muito importante na organização, tendo impacto não só no desempenho, mas, também, estando diretamente ligado à tomada de decisão, à cultura, às definições estratégicas e à criação e armazenamento de conhecimento.

A perspectiva de que os gêneros possam intervir no desempenho da organização também permeia a relação entre a estética e o desempenho organizacional, uma vez que, segundo Leal (2002), a estética é onipresente, influenciando as ações da organização por meio de perspectivas subjetivas. Devido a isso, a estética se coloca como uma das formas possíveis para a análise organizacional, que pode dar conta tanto do entendimento dos produtos organizacionais quanto do espaço de inserção dos trabalhadores. Todavia, Leal (2002) considera ser importante que a estética também seja utilizada para compreender como os processos de aprendizagem e conhecimento ocorrem e são influenciados nas organizações.

A estética organizacional pode auxiliar na geração de conhecimento, uma vez que, segundo Taylor e Hansen (2005), o conhecimento sensorial decorrente da estética auxilia o indivíduo a construir sua apreensão do mundo, bem como envolve diretamente seus pensamentos e percepções, que, depois, podem influenciar novas percepções estéticas. Para Taylor e Hansen (2005), o conhecimento estético pode gerar novas ideias e desencadear atenções dos indivíduos para determinados aspectos, ao mesmo tempo que sua tradução para palavras ou textos é difícil e complexa. Embora esse tipo de conhecimento sempre exista nas organizações, a sua gestão parece ser relegada a um segundo plano, quando comparada à gestão e compreensão dos conhecimentos intelectuais ou explícitos propiciados pela e na organização. Nesse sentido, diferentes apreensões de conhecimento estético e também da importância despendida a determinados aspectos podem ser compreendidas pelas diferenças de gêneros, uma vez que, segundo Freedman e Phillips (1988, p. 237) os padrões de valores e a motivação para o trabalho são diferentes entre os gêneros, sendo que diferentes razões podem ser percebidas para estar, agir e reagir no ambiente de trabalho, pois as "mulheres podem desenvolver necessidades e motivações distintas e, além disso, respostas únicas às características que configuram o trabalho". Essa visão é compartilhada por Shaw e Cassell (2007) que afirmam que diferentes processos de socialização são as principais causas para as distintas motivações que levam a adentrar e permanecer na esfera do trabalho. 
Essas diferenças de gênero também são compatíveis com as diferenças encontradas para a apreensão de conhecimento sensorial. Segundo Taylor e Hansen (2005), o conhecimento sensorial, proveniente das compreensões estéticas, pode interferir nas cognições humanas, assim como pode ser significativo para alterar e constituir o modo de agir de um indivíduo, moldando como suas ações são expressas no ambiente. Assim, percepções estéticas que podem ser distintas entre os gêneros têm capacidade de conduzir a processos cognitivos distintos, ou seja, a forma e como a estética da organização é percebida por cada gênero pode moldar suas ações, bem como intervir nas alterações e concepções que cada gênero possui do ambiente onde está inserido.

Além disso, o conhecimento tácito de cada gênero pode ser a fonte dos discursos sobre a estética da organização (MACK, 2007), assim como os próprios lugares de trabalho podem agir como ativadores das histórias e dos discursos que revelam a subjetividade inerente aos artefatos constantes na organização e que compõem sua estética, por isso, a compreensão do conhecimento apreendido da estética organizacional deve ser verificado no local em que ele foi adquirido, para que a profundidade da análise sobre este seja atingida, visto que os artefatos podem ativar e interagir com o indivíduo, revelando o conhecimento de forma mais completa.

Baseado no exposto, apresenta-se a seguinte sugestão de estudo:

\section{Sugestão 5: A interação entre os conhecimentos sobre estética organizacional e sobre as relações de gênero na organização pode oferecer novas perspectivas para a compreensão da influência do conhecimento no desempenho organizacional.}

Em outra linha de ligação com o desempenho organizacional, Herring (2009) identificou uma possível relação entre a diversidade de gênero na organização e o aumento da receita de vendas, do número de consumidores, do market share e da lucratividade relativa. Segundo Herring (2009), tais incrementos de desempenho podem ser provenientes de uma espécie de conflito criativo, advindo da diversidade, sendo que os conflitos atuariam como forças direcionadoras para a busca de soluções de forma mais ágil e rápida, além disso, o conflito pode levar à contestação, à geração de novas ideias, a uma maior criatividade na organização e ao aumento da necessidade de comunicação. Nesse aspecto, Gagliardi (2001) cita que a experiência estética que os indivíduos possuem na organização é uma forma de comunicação, que ocorre ou ganha força à medida que os indivíduos vão tomando conhecimento sensorial sobre os artefatos ou decorrências destes. Em outras palavras, à medida que o indivíduo vai tendo um maior número de sensações do ambiente, ele, com o tempo, vai se tornando mais apto a criar sentido para o ambiente que, por consequência, será por ele encarado diariamente no exercício de suas atividades laborais.

A comunicação entre os gêneros por meio dos artefatos também pode ser investigada sob o objetivo de compreender de forma mais aprofundada como o desempenho organizacional é afetado, não só pela diversidade de gêneros, mas, também, pelos diversos usos dos artefatos estéticos que cada gênero pode fazer no ambiente de trabalho. Além disso, os artefatos também podem atuar como uma forma de construção de canais mais ágeis de comunicação entre os indivíduos, o que pode intervir nas relações criativas e conflitantes entre os trabalhadores, propiciando (ou não) a alavancagem de desempenho da organização.

Nesse sentido, segundo Hackett, Mirvis e Sales (1991), os gêneros também podem ser diferentes nos padrões de adoção de novas tecnologias no ambiente de trabalho, o que pode intervir na comunicação e criatividade dos indivíduos. No mesmo estudo, foi evidenciado que percepções acerca de novas tecnologias também podem impactar percepções distintas sobre a melhora (piora) do espaço de trabalho. Menezes e Diniz (2011) também evidenciam que os atributos evidenciados pelos gêneros e, consequentemente valorizados pelas organizações, podem ser diferentes, favorecendo a conquista de espaços distintos e a criação de simbolismos diferentes. 
Diante do exposto, apresenta-se a seguinte sugestão de estudo:

\section{Sugestão 6: Juntos, os conhecimentos sobre a estética organizacional e sobre as relações de gênero podem fornecer uma visão mais ampla e profunda sobre a influência da comunicação no desempenho organizacional.}

Baseado no exposto, sugere-se que a investigação das relações entre os gêneros e a estética organizacional atente para as formas de geração de conhecimento e, também, de comunicação como aspectos que podem ter relevante impacto no desempenho organizacional, sendo que, a compreensão de como os gêneros e a estética atuam conjuntamente pode conduzir a interpretações mais aprofundadas sobre a organização no que tange ao desempenho, inferindo especificamente sobre as formas de comunicação e conhecimento.

\section{Implicações Finais}

Este ensaio teórico observou duas temáticas que vêm sendo estudadas e que merecem atenção dentro dos Estudos Organizacionais, os estudos das relações de gênero nas organizações e também os estudos sobre estética organizacional, sendo que o enfoque adotado buscou elucidar possíveis intersecções entre os estudos para pesquisas futuras sobre as relações entre os indivíduos e a organização, a cultura organizacional e o desempenho organizacional.

Primeiramente, foram analisadas as ligações entre os estudos das características de gêneros nas organizações e a estética organizacional sob o enfoque das conexões entre os indivíduos e a organização. Diante desse tópico, coloca-se que a compreensão conjunta da interação dos gêneros com a estética organizacional pode ser relevante para um maior conhecimento dos reais motivos pelos quais os indivíduos permanecem nas organizações, garantindo, ao menos, uma nova visão para a temática, uma vez que em ambas as literaturas essa temática parece ser tangenciada. Com isso, suscita-se que a junção possa trazer benefícios ao entendimento das conexões entre indivíduos e organização.

Em um segundo momento, foram colocadas em pauta as interligações entre estética e gênero que podem contribuir para o entendimento da cultura organizacional, já que esse tópico também é citado em diferentes estudos sobre gênero e sobre estética, separadamente, por isso a junção proposta visa a instigar os pesquisadores a considerar em seus futuros estudos tanto as relações entre os gêneros quanto a estética da organização como variáveis que podem dar uma maior sustentação às explicações sobre a cultura da organização. Convém ressaltar que enfoques sobre a manutenção ou alteração dos simbolismos e habitus masculino no ambiente organizacional pode ser uma vertente importante para o desenvolvimento de novos estudos que contemplem as relações de gênero e estética organizacional. Além disso, a visão cultural sobre os gêneros e sobre a estética pode ser um aspecto que une os dois tópicos, provando que estudos inversos (sobre o aculturamento dos gêneros na organização e a cultura sobre os artefatos) também podem ser realizados.

Por fim, discute-se como a comunicação e o conhecimento podem ser estudados junto à interação entre os gêneros e a estética organizacional, a fim de prover maiores entendimentos sobre o desempenho das organizações. As comunicações podem sofrer o impacto das relações de gênero bem como ser interpeladas pelos sentidos e usos dos artefatos organizacionais, o que também parece ser verdadeiro para a geração de conhecimento tácito, que pode ser apreendido dos espaços organizacionais e que pode ter diferentes conotações entre os gêneros. 
Baseado no exposto, Alvesson e Billing (1992) colocam que os estudos das relações de gênero nas organizações podem ter amplas variações, por isso, as sugestões e ideias de estudos futuros suscitadas neste ensaio devem ser compreendidas com atenção e ajustadas a cada contexto de observação, ao invés de ser compreendidas como verdades absolutas. Essa assunção também é verdadeira para os estudos sobre estética organizacional. No entanto, não assumindo uma postura relativista e em concordância com Alvesson e Billing (1992), certos padrões podem ser encontrados com a constância da pesquisa e do cuidado com as demais variáveis que podem intervir na compreensão do fenômeno.

Salienta-se que este estudo abre perspectivas de análise para além das interações entre os temas gênero e estética organizacional. Outros temas, como as relações de poder, os conflitos e a cooperação, podem ser explorados a partir da literatura sobre estética organizacional e da literatura de relações de gênero.

\section{Referências}

ALVESSON, M.; BILLING, Y. D. Gender and organization: towards a differentiated understanding. Organization Studies, v. 13, n. 12, p. 73-102, 1992.

ANDRADE, A. L. S. et al. Gênero nas organizações: um estudo no setor bancário. Revista de Administração de Empresas, v. 1, n. 1, p. 1-15, 2002.

BOURDIEU, P. A Dominação masculina. Rio de Janeiro: Bertrand, 1999.

CAIRNS, G. Aesthetics, morality and power: design as espoused freedom and implicit control. Human Relations, v. 55, n. 7, p. 799-820, 2002.

CALÁS, M. B.; SMIRCICH, L. Do ponto de vista da mulher: abordagens feministas nos estudos organizacionais. In: CLEGG, S.; HARDY, C; NORD, D. (Org.). Handbook de estudos organizacionais. v. 1. São Paulo: Atlas, 1999. 275$329 \mathrm{p}$.

CAPPELLE, M. C. A.; NETTO, L. C. A. P. Relações de gênero na polícia: a construção das representações sociais do masculino e do feminino em uma organização militar. In: ENCONTRO NACIONAL DA ASSOCIAÇÃO DE PÓSGRADUAÇÃO EM ADMINISTRAÇÃO, 25., 2001, Rio de Janeiro. Anais... Rio de Janeiro: Anpad, 2001, 1-15 p.

. et al. A produção científica sobre gênero na administração: uma meta-análise. In: ENCONTRO NACIONAL DA ASSOCIAÇÃO DE PÓS-GRADUAÇÃ̃O EM ADMINISTRAÇÃO, 30., 2006, Salvador. Anais... Rio de Janeiro: Anpad, 2006. 1-17 p.

CHARLESWORTH, S.; BAIRD, M. Getting gender on the agenda: the tale of two organizations. Women in Management Review, v. 22, n. 5, p. 391-404, 2007.

CORRÊA, A. M. H. et al. Soldadinhos-de-chumbo e bonecas: representações sociais do masculino e feminino em jornais de empresas. Revista de Administração Contemporânea, v. 11, n. 2, p. 191-211, 2007.

COX, J. W.; MINAHAN, S. Organization, decoration. Organization, v. 12, n. 4, p. 529-548, 2005.

DESHPANDE, R.; WEBSTER, F. E. Organizational culture and marketing: defining the research. Journal of Marketing, v. 53, n. 1, p. 3-15, 1989.

ECCEL, C. S. Estudos de gênero nas organizações: implicações teórico-metodológicas. In: ENCONTRO NACIONAL DA ASSOCIAÇÃO DE PÓS-GRADUAÇÃO EM ADMINISTRAÇÃO, 34., 2010, Rio de Janeiro. Anais... Rio de Janeiro: Anpad, 2010. 1-15 p. 
FOURNIER, V.; SMITH, W. Scripting masculinity. Ephemera: Theory \& Politics in Organization, v. 6, n. 2, p. 141162,2006

FREEDMAN, S. M.; PHILLIPS, J. S. The changing nature of research on women at work. Journal of Management, v. 14, n. 2, p. 231-251, 1988.

GAGLIARDI, P. Explorando o lado estético da vida organizacional. In: CLEGG, S.; HARDY, C.; NORD, W. R. (Org.). Handbook de Estudos Organizacionais. v. 2. São Paulo: Atlas, 2001. 127-149 p.

GIBB, S. Imagination, creativity, and HRB: an aesthetic perspective. Human Resource Development Review, v. 3 , n. 1, p. 53-74, 2004.

HACKETT, E. J.; MIRVIS, P. H.; SALES, A. L. Women's and men's expectations about the effects of new technology at work. Group Organization Management, v. 16, n. 1, p. 60-85, 1991.

HERRING, C. Does diversity pay? Race, gender, and the business case for diversity. American Sociological Review, v. 74, n. 2, p. 208-224, 2009.

HOLVINO, E. Intersections: the simultaneity of race, gender and class in organization studies. Gender, Work and Organization, v. 17, n. 3, p. 248-277, 2010.

LEAL, R. S. Razão, práxis e estética: integração de diferentes dimensões de análise organizacional. In: ENCONTRO NACIONAL DA ASSOCIAÇÃO DE ENTIDADES DE PÓS-GRADUAÇÃO EM ADMINISTRAÇÃO, 26., 2002 , Salvador. Anais... Salvador: Anpad, 2002. 1-13 p.

LEDET, L. M.; HENLEY, T. B. Perceptions of women's power as a function of position within an organization. The Journal of Psychology, v. 134, n. 5, p. 515-526, 2000.

MACK, K. S. Senses of seascapes: aesthetic and the passion for knowledge. Organization, v. 14, n. 3, p. 373-390, 2007.

MARTIN, P. Y. Sensations, bodies, and the 'spirit of a place': aesthetics in residential organizations for the elderly. Human Relations, v. 55, n. 7, p. 861-885, 2002.

MATHIEU, C. Practising gender in organizations: the critical gap between practical and discursive consciousness. Management Learning, v. 40, n. 2, p. 177-193, 2009.

MENEZES, R. S. S.; DINIZ, A. P. R. Simbolismos de gênero e trabalho: uma análise das feminilidades na gestão. In: ENCONTRO NACIONAL DA ASSOCIAÇÃO DE PÓS-GRADUAÇÃO EM ADMINISTRAÇÃO, 35., 2011, Rio de Janeiro. Anais... Rio de Janeiro: Anpad, 2011. 1-17 p.

MORGAN, G. Imagens da organização. São Paulo: Atlas, 1996.

MURGIA, A.; POGGIO, B. Challenging hegemonic masculinities: men's stories on gender culture in organizations. Organization, v. 16, n. 3, p. 407-423, 2009.

RAFAELI, A.; VILNAI-YAVETS, I. Emotion as a connection of physical artifacts and organizations. Organization Science, v. 15, n. 6, p. 671-686, 2004.

SCOTT, J. W. Gênero: uma categoria útil de análise histórica. Educação \& Realidade, v. 20, n. 2, p. 71-99, 1995.

SHAW, S.; CASSELL, C. "That's not how I see it": female and male perspectives on the academic role. Women in Management Review, v. 22, n. 6, p. 497-515, 2007.

SMIRCICH, L. Concepts of culture and organizational. Administrative Science Quarterly, v. 28, n. 3, p. 339-358, 1983. 
STRATI, A. Aesthetic understanding of organizational life. The Academy of Management Review, v. 17, n. 3, p. 568581, 1992.

TAYLOR, S. S.; HANSEN, H. Finding form: looking at the field of organizational aesthetics. Journal of Management Studies, v. 42, n. 6, p. 1211-1231, 2005.

TAYLOR, S.; SPICER, A. Time for space: a narrative review of research on organizational spaces. International Journal of Management Reviews, v. 9, n. 4, p. 325-346, 2007.

TIESSEN, R. Re-inventing the gendered organization: staff attitudes towards women and gender mainstreaming in NGOs in Malawi. Gender, Work and Organization, v. 11, n. 6, p. 689-708, 2004.

TYLER, M.; COHEN, L. Spaces that matter: gender performativity and organizational space. Organization Studies, v. 31, n. 2, p. 175-198, 2010.

WEGGEMAN, M.; LAMMERS, I.; AKKERMANS, H. Aesthetics from a design perspective. Journal of Organizational Change Management, v. 20, n. 3, p. 346-358, 2007.

WEICK, K. E. Sensemaking in organization. London: Sage, 1995.

WITZ, A.; WARHURST, C.; NICKSON, D. The labour of aesthetic and the aesthetic of organization. Organization, v. 10 , n. 1, p. 33-54, 2003 